\title{
A Moving Body Recognition Method with Background Robustness
}

\author{
Shoujia WANG ${ }^{a}$, *Wenhui $\mathrm{LI}^{\mathrm{b}}$, Hongyin NI, Bo FU and Jinlong ZHU \\ College of Computer Science and Technology, Jilin University, Changchun, China 130012 \\ awangsj035@163.com, *liwh@jlu.edu.cn
}

Keywords: Body recognition; tripling temporal difference; mathematical morphology; quadruple directions connection.

\begin{abstract}
Nowadays, moving body recognition method is used widely in all kinds of videos. But recognition accuracy of these methods is changed negatively because of complexity of background, e.g. void and noise. In this paper, we put forward a new recognition method with background robustness. Firstly, we get moving body by tripling temporal difference method. Then we eliminate noise of these images by mathematical morphology. Finally, we connect disconnected areas with quadruple directions connection method. The new method is more accurate and less computational in real time experiment by used less computation. The experiment result also shows its robustness of background.
\end{abstract}

\section{Introduction}

With development of information technology, recognition of moving body becomes an important research area in military affairs, national defense and other domains. Usually, we recognize moving body continuously in video supervisory control. The mostly used methods are background subtraction method [1], optical flow method [2] and temporal difference method [3]. But all of them exists problems.

A pixel model of background must be constructed when to use background subtraction method. Then it get moving object by compared every frame to background. Though it is simple and quick, its accuracy is not well by change of illumination, noise and so on. The main problems are elongation phenomenon of void, shadow and moving object.

The main task of optical method is computation of optic flow filed. It contains two steps. One is to estimate motion filed by time-space gradient of image sequence under smooth restrain condition, the other is to divide moving object and background by change of motion filed. The main problem is the high computation complexity. So it cannot be used in real-time domain by its high computational time and weak antinoise performance [4].

Temporal difference method uses difference of neighbor frame to recognize. It recognizes moving object when difference result is larger than a given threshold. This method shows robustness of illumination and shadow [5]. So we get foreground with an improved tripling temporal difference method firstly. Then we smooth edge and eliminate noise of these images by mathematical morphology. Finally, we eliminate void and connect disconnected areas with quadruple directions connection method. The experiment result validate that the new method is fast and accurate. Moreover, this method shows its robustness of background.

\section{Recognition method of moving body}

\section{Extraction prospects method}

The temporal difference method subtracts all pixels of neighbor frames. It is considered to be static when difference is small by similar illumination. Oppositely, moving object effects large difference. Then we sign these areas with large difference and find moving object in frames [6].

We show itsformula below.

$$
\mathrm{G}_{i, i-1}(x, y)=\left|X_{i}(x, y)-X_{i-1}(x-y)\right|
$$




$$
B_{i, i-1}(x, y)= \begin{cases}1 & \text { if } G_{i, i-1}(x, y) \geq t h \\ 0 & \text { if } G_{i, i-1}(x, y)<t h\end{cases}
$$

Temporal difference method shows well performance when movement is uniform motion. But it is not so well when movement is not uniform motion. Another problem is that moving object contains large pixels when it is recognized by temporal difference method. So we improved it to tripling temporal difference method to recognize moving body. This method not only improve recognition rate, but also improve integrality of moving object. To let $I_{n}(x)$ to be pixel gray of fore-frame and $T_{n}(x)$ to be threshold, we consider pixels $\mathrm{x}$ are moving when they fit the formula below.

$$
\begin{aligned}
& \left|I_{n}(x)-I_{n-1}(x)\right|>T_{n}(x) \\
& \left|I_{n}(x)-I_{n-2}(x)\right|>T_{n}(x)
\end{aligned}
$$

Figure 1 is algorithm flow chart of tripling temporal difference method.

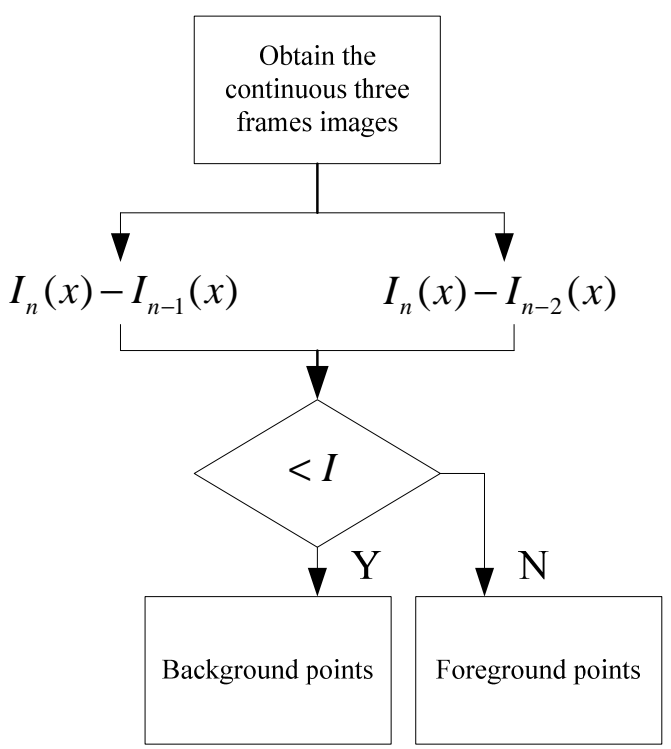

Fig.1 Flow chart of tripling temporal difference method

\section{Quadruple directions connection process}

After dilatation, edge of foreground object is clearer and void in object is partly filled. Then we use quadruple directions connection method to connect remaining void. Origin image is shown in figure 2 with foreground color black. Figure 3 is target area of connection by use connection model. In this paper, the connection distance is 5 pixels. Quadruple directions means left, upper left, upper and upper right. We don't connect the other four directions because that they are symmetrical. In fact, quadruple direction methods can be reduced half computation.

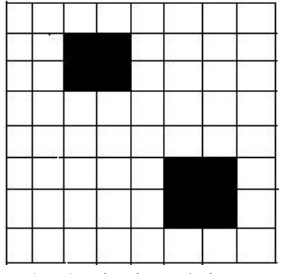

Fig.2 Original image

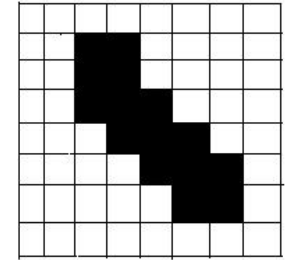

Fig.3 Process image

\section{Experiment}

We use Inria database[7] to process our experiment, which is a harder passenger database created by Dalal and based on Mit database. It is standard test database in this study area. We use 1000 positive samples and 1000 negative samples to test.

We show our flow chart in figure 4. 


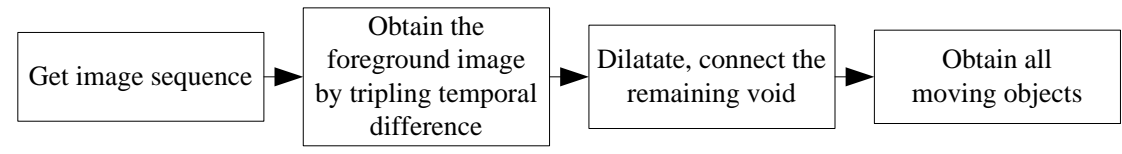

Fig.4 Flow chart of moving object capture

The steps of experiment algorithm are shown below.

Step1. To catch 3 continual image frames $I_{n}, I_{(n-1)}, I_{(n-2)}$.

Step2. For all pixels $\mathrm{x}$ in frame, compute two difference $\operatorname{diff} f_{1}=\mathrm{I}_{\mathrm{n}}(x)-I_{(n-1)}(x)$ and $\operatorname{diff}_{2}=\mathrm{I}_{\mathrm{n}}(x)-I_{(n-2)}(x)$

Step 3. To set a threshold $h$ (we use a static $h=25.0$ ). If $\operatorname{diff}_{1}<h$ and $\operatorname{diff}_{2}<h$, to set the pixel in foreground, otherwise, to set it in background.

Step 4. Dilate the binary image.

Step 5. Use quadruple direction connection method to connect the disconnection area.

Figure 5a, 5b and 5c are three continuous frame images, figure 6 are the contrast diagrams in different detection methods, those are temporal difference method in figure 6a, tripling temporal difference method in figure $6 \mathrm{~b}$ and this paper's method in figure 6c. From the experiment results, we can see the method in in figure6c has better effect than other two method in figure 6a and figure 6b.This method not only eliminate the noise around, but also make the moving body outline more clearly, moreover, it remove the void effectively. We can see from figure 6 that the method in this paper could enhance the recognition accuracy of moving body in complex background.

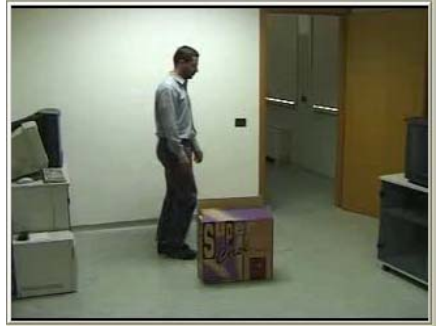

a

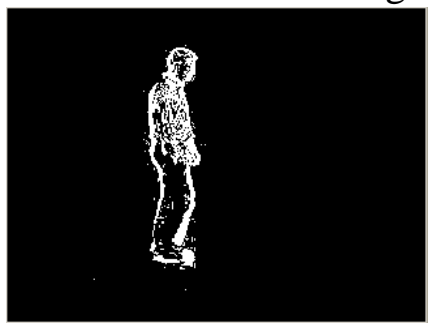

a

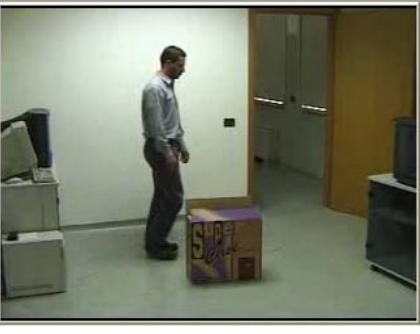

b

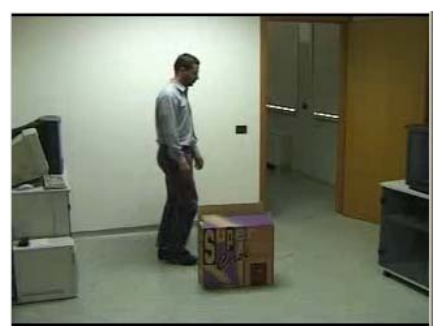

C

Fig.5 Three continuous frame images

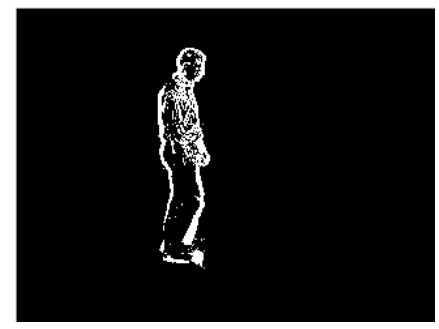

b

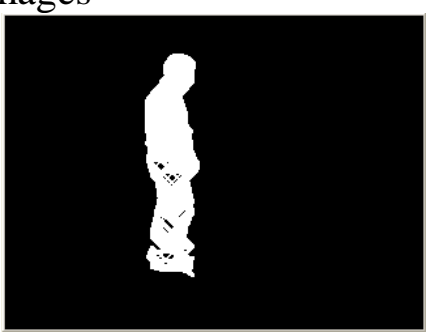

C

Fig.6 The contrast diagram in three recognition methods

\section{Conclusion}

This paper puts forward a new moving object recognition method. In this paper, we use tripling temporal difference method to recognize moving object and dilate the computed binary image with mathematical morphology. At last, we use quadruple directions connection method to connect disconnection areas. This method not only needs no restraint of background, but also shows less computation and higher accurate. Then with large experiments, experiment results also show its positive computational complexity, accuracy and robustness.

\section{Acknowledgments}

This work is supported by the grants from "National Natural Science Foundation of China" [No. 60873147]. 


\section{References}

[1] S Gupt, O Masound, R F K Martin, N P Papanikolopoulos: Detection and classification for vehicles. Intelligent Transportation Systems. Vol. 3-1 (2002), p. 37-47.

[2] Yuqiang Fang, Bin Dai, in: An Improved Moving Target Detecting and Tracking Based On Optical Flow Technique and Kalman Filter, Progress in the 4th International Conference on Computer Science \& Education, (2009).

[3] RT Collins , A Lipton , T Kanade: A system for video surveillance and monitoring:VSAM final report, The Robotics Institute , Carnegie Mellon University , Pittsburgh PA , (2000) .

[4] WANG Xiaoyan ,ZHANG Yanzhu ,DONG Huiying ,LI Yuan ,LI Xiaojuan: Algorithm Research on Three-Frame Difference for Detection of Moving Target. Journal of Shenyang Ligong University. Vol. 30-6 (2011), p. 82-85.

[5] Lin Rui , Du Zhijiang , Sun Lining, in: Moving Object Tracking based on Mobile Robot Vision, Progress in International Conference on Mechatronics and Automation , (2009).

[6] GY Chen , Buitd, A Krzyzak,in: Image denoising using neighbouring wavelet coefficients, Progress in IEEE International Conference on Acoustics Speech , and Signal Processing(ICASSP) , (2004).

[7] ZENG Chun, LI Xiao-hua, ZHOU Ji-liu: Pedestrian detection based on HOG of ROI. Computer Engineering. Vol. 35-24 (2009), p. 182-184. 